\title{
Estudo termodinâmico da adsorção de cobre (II) em montmorilonita organicamente modificada
}

\section{(Thermodynamic study of the adsorption of copper (II) onto organic-modified montmorillonite)}

\author{
W. L. L. da Silva ${ }^{1 *}$, J. de A.Simoni ${ }^{1}$ \\ ${ }^{1}$ Universidade de Campinas, Cid. Univ. “Zeferino Vaz”, 13083-970, Campinas, SP, Brasil
}

\begin{abstract}
Resumo
O presente estudo teve como objetivo avaliar a termodinâmica e o equilíbrio de adsorção, em solução aquosa, do íon $\mathrm{Cu}^{2+}$ em uma montmorilonita organofuncionalizada por 3-aminopropiltrietoxissilano. Esse adsorvente foi preparado pela intercalação inicial de brometo de cetiltrimetilamônio e depois a funcionalização com o 3-aminopropiltrietoxissilano. A organofuncionalização ocorreu na superfície externa da matriz inorgânica. A capacidade máxima de adsorção de $\mathrm{Cu}^{2+}$ pela amostra organofuncionalizada, prevista pela densidade de imobilização $\left(1,46.10^{-3} \mathrm{~mol} . \mathrm{g}^{-1}\right)$ foi de cerca de $92 \mathrm{mg}$ de $\mathrm{Cu}^{2+}$ por grama de argila. A variação da entalpia na adsorção $(\Delta \mathrm{H})$ obtida por via calorimétrica foi de $-1,4 \pm 0,3 \mathrm{~kJ} \cdot \mathrm{mol}^{-1}$, valor muito pequeno para ser determinado por aplicação de método da segunda lei. Tomando por base o modelo de Langmuir, com modificações, obtiveram-se valores de constantes de afinidade de $47 \pm 10,41 \pm 9$ e $48 \pm 6 \mathrm{dm}^{3} . \mathrm{mol}^{-1}$, para as temperaturas de 25,35 e $45^{\circ} \mathrm{C}$, respectivamente, o que evidenciou grandezas iguais dentro das incertezas experimentais.
\end{abstract}

Palavras-chave: termodinâmica, montmorilonita, adsorção, 3-aminopropiltrietoxissilano, cobre.

\section{Abstract}

The aim of present study was to evaluate thermodynamics and the equilibrium of adsorption, in aqueous solution, of the Cu ${ }^{2+}$ ion in a montmorillonite organofunctionalized by 3-aminopropyltriethoxysilane. This adsorbent was prepared by the initial intercalation of cetyltrimethylammonium bromide and then functionalization by 3-aminopropyltriethoxysilane. The organofunctionalization occurred on the outer surface of the inorganic matrix. The maximum adsorption capacity of $\mathrm{Cu}^{2+}$ by the organofunctionalized sample, predicted by the immobilization density $\left(1.46 \times 10^{-3} \mathrm{~mol} . \mathrm{g}^{-1}\right)$, was about $92 \mathrm{mg}$ of $\mathrm{Cu}^{2+}$ per gram of clay. The adsorption enthalpy change $(\Delta H)$, obtained by calorimetric method was $-1.4 \pm 0.3 \mathrm{~kJ}^{\mathrm{mol}} \mathrm{m}^{-1}$, a very small value to be determined by the application of

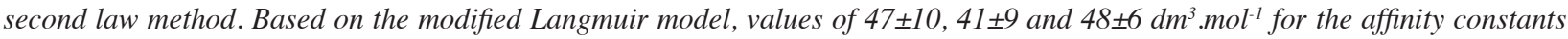
were obtained for temperatures of 25,35 and $45{ }^{\circ} \mathrm{C}$, respectively, which showed equal magnitudes for these physical quantities within the experimental uncertainties.

Keywords: thermodynamics, montmorillonite, adsorption, 3-aminopropyltriethoxysilane, copper.

\section{INTRODUÇÃO}

Oatual crescimento da exploração e consumode matériasprimas, juntamente com o crescente aumento da população, vem causando grandes modificações no meio ambiente. Entre outras coisas, esse panorama também contribui para o aumento expressivo da contaminação do solo e dos efluentes por íons de metais pesados. Os metais pesados são tóxicos, não biodegradáveis e persistentes, e gradualmente se acumulam nos tecidos vivos de toda a cadeia alimentar. Alguns métodos convencionais de tratamento de efluentes contendo metais pesados, como precipitação, oxidação/ redução, filtração, troca iônica, eletroquímica, dentre outros, são muitas vezes restritos por inviabilidade técnica e, às vezes, até econômica. Nesse sentido, a adsorção é considerada um método efetivo e barato, quando comparado

*weber.silva@iqm.unicamp.br aos demais $[1,2]$.

O sólido mais empregado comercialmente no tratamento de efluentes por adsorção é o carvão ativado, entretanto seu uso tem certas limitações, e seu custo não é baixo [2]. Deste modo, a busca por novos materiais de baixo custo, que possam substituir o carvão ativado, é uma preocupação constante. O interesse pelo uso de argilominerais como adsorvente nesse tipo de tratamento vem se acentuando $[3,4]$. A montmorilonita é um argilomineral com estrutura lamelar composta por duas camadas tetraédricas apontando para dentro e com uma camada octaédrica de alumínio central [5]. Alguns atributos da montmorilonita são a sua alta capacidade de troca catiônica, a propriedade de intumescimento e áreas superficiais elevadas, que fazem com que a mesma seja amplamente utilizada em várias aplicações, sendo umas delas como adsorvente [6]. Nesse sentido, o presente estudo avalia a capacidade de adsorção e o estudo termodinâmico da adsorção de cobre (II) em solução 
aquosa por uma montmorilonita organicamente modificada. A montmorilonita foi modificada por intercalação inicial com o surfactante CTAB e, em seguida, com o 3-aminopropiltrietoxissilano. O tratamento matemático dos dados do equilíbrio de adsorção foi realizado por ajustes matemáticos não lineares, baseados nos modelos de Langmuir, Freundlich e Sips. A termodinâmica da adsorção de cobre (II) na montmorilonita organofuncionalizada é discutida em termos de grandezas como a constante de afinidade de Langmuir e variação de entalpia $(\Delta \mathrm{H})$, obtida via calorimetria isotérmica.

\section{MATERIAS E MÉTODOS}

Preparação da argila: a argila (MMT) foi proveniente do município de Campina Grande (latitude Sul $7^{\circ} 13^{\prime}$ e longitude Oeste $35^{\circ} 52^{\prime}$, no estado da Paraíba) e composta majoritariamente pelo argilomineral montmorilonita. O método utilizado para remoção das impurezas orgânicas e inorgânicas foi realizado de acordo com a literatura [7, 8]. Remoção da matéria orgânica: cerca de $500 \mathrm{~g}$ da argila natural foram triturados num moinho de bolas, em seguida passados por uma peneira granulométrica (Granutest), com abertura de malha de $0,200 \mathrm{~mm}$. Posteriormente a argila peneirada foi suspensa em $750 \mathrm{~mL}$ de água oxigenada 15 volumes. $\mathrm{O} \mathrm{pH}$ da solução foi mantido constante por tamponamento com ácido acético/acetato em pH igual a 4,7. A suspensão formada foi mantida sob agitação mecânica e aquecimento a $50{ }^{\circ} \mathrm{C}$, durante $72 \mathrm{~h}$. Em seguida a suspensão foi deixada em repouso para a decantação do sólido, permitindo assim a separação das fases líquida e sólida. A fase líquida foi removida por sifonação e a fase sólida foi lavada com 1,5 L de água destilada; a suspensão resultante dessa lavagem foi deixada em repouso até a separação das fases por decantação. Esse processo de lavagem da fase sólida foi realizado três vezes para garantir a remoção completa dos resíduos em solução. Remoção de óxidos de ferro: após a remoção da matéria orgânica, a argila resultante foi suspensa em 1,5 L de solução 0,15 mol. $\mathrm{L}^{-1}$ de ácido clorídrico, e mantida sob agitação mecânica a $40^{\circ} \mathrm{C}$ por $2 \mathrm{~h}$. Em seguida, foram adicionados $2,0 \mathrm{~g}$ de cloreto de sódio, e a suspensão permaneceu em repouso até decantação do material sólido. Após sifonação da solução sobrenadante, adicionou-se ao material sólido cerca de 1,5 L de solução aquosa de ácido cítrico de concentração $10 \%$. A suspensão foi mantida sob agitação mecânica à temperatura constante de $40{ }^{\circ} \mathrm{C}$, por 2 h. Os dois procedimentos, ácido clorídrico e ácido cítrico foram repetidos outras duas vezes. O sólido resultante foi lavado com água destilada até o teste negativo para cloreto utilizando-se solução de nitrato de prata. Remoção de areia e quartzo: a argila, livre de impurezas orgânicas e óxidos de ferro, foi suspensa em água destilada e introduzida em sacos plásticos adaptados aos tubos da centrífuga, promovendose a centrifugação a $3000 \mathrm{rpm}$ até a separação total das fases. A fase líquida foi separada e as fases sólidas, argila e óxidos de silício, foram separadas fisicamente; a argila é menos densa e por isso permaneceu na parte superior do sólido centrifugado, dentro do saco plástico. A separação das fases sólidas foi feita mecanicamente, utilizando-se como referência as diferenças de coloração entre os óxidos e a argila. Esse processo de separação mecânica (física) foi repetido três vezes, como forma de garantir uma separação mais efetiva.

Preparação da argila monoiônica em sódio: a argila obtida após os procedimentos anteriores encontrava-se na forma ácida (MMT-H), pois o processo de eliminação do óxido de ferro foi feito em meio ácido. Um processo de troca iônica para tornar a argila monoiônica em sódio foi feito pelo tratamento da argila (MMT-H) com cerca de $1 \mathrm{~L}$ de uma solução aquosa de cloreto de sódio de concentração 1 mol.L $L^{-1}$. A suspensão foi mantida sob agitação mecânica por $12 \mathrm{~h}$, à temperatura constante de $40{ }^{\circ} \mathrm{C}$. Após este período, a suspensão foi centrifugada até a separação total das fases, desprezando-se a fase líquida. O processo de troca iônica foi repetido por mais duas vezes e argila na sua forma sódica foi lavada com água destilada até teste negativo para cloreto. Em seguida, o material resultante (MMT-Na) foi seco em estufa a $40^{\circ} \mathrm{C}$, por $24 \mathrm{~h}$. Intercalação com CTAB: a montmorilonita (MMT-Na) foi submetida a um processo de intercalação com brometo de cetiltrimetilamônio (CTAB). Para esta intercalação, $10 \mathrm{~g}$ de MMT-Na e $5 \mathrm{~g}$ de CTAB foram dispersos em $500 \mathrm{~mL}$ de água deionizada e o sistema foi mantido sob agitação por $5 \mathrm{~h}$ à temperatura ambiente. $\mathrm{O}$ sólido resultante foi retirado por filtração e lavado com água deionizada por várias vezes até se tornar livre do $\mathrm{CTAB}$ em excesso. O material sólido foi seco em estufa a $80^{\circ} \mathrm{C}$ por 24 $\mathrm{h}$, resultando na amostra MMT-CTAB [9].

Procedimento de organofuncionalização: a montmorilonita intercalada com CTAB (MMT-CTAB) foi submetida ao processo de organofuncionalização com 3-aminopropiltrietoxissilano (APTES). Em suma, $10 \mathrm{~g}$ de MMT-CTAB foram suspensos em $300 \mathrm{~mL}$ de tolueno num balão de fundo redondo de três bocas, equipado com um termômetro, um condensador de refluxo e uma entrada de nitrogênio gasoso. Adicionaram-se, então, 3,2 mL de água deionizada à suspensão e a mistura foi agitada por $2 \mathrm{~h}$ à temperatura ambiente para permitir que a água se dispersasse através do material, tornando sua superfície totalmente hidroxilada. Após esse processo, $17 \mathrm{~mL}$ do organossilano foram adicionados à mistura, que posteriormente foi deixada em refluxo por $24 \mathrm{~h}$ a $80{ }^{\circ} \mathrm{C}$ [10]. Após este procedimento, o sólido obtido (MMT-CTAB-APTES) foi separado por decantação e lavado com água deionizada, tolueno e com uma solução etanol/ácido clorídrico $0,1 \mathrm{~mol} . \mathrm{L}^{-1}$ na proporção 1:1, em seguida, filtrado e seco a $80^{\circ} \mathrm{C}$ [11]. Procedimento da remoção do cátion $C T A^{+}$: o material resultante da organofuncionalização (MMT-CTAB-APTES) foi suspenso em $250 \mathrm{~mL}$ de solução de cloreto de sódio 0,1 mol. $\mathrm{L}^{-1}$ e a suspensão foi agitada por $3 \mathrm{~h}$ à temperatura ambiente. $\mathrm{O}$ sólido foi filtrado e lavado com água deionizada por várias vezes. Esse procedimento foi necessário para a remoção do $\mathrm{CTA}^{+}$intercalado. A completude da remoção foi verificada e confirmada por difração de raios X. O material obtido foi denominado de MMT-APTES [11]. 
Métodos de caracterização dos materiais obtidos. Difratometria de raios $X(D R X)$ : os difratogramas de raios $\mathrm{X}$ no pó foram obtidos em um difratômetro (Shimadzu, XRD7000), com radiação $\mathrm{CuK} \alpha(\lambda=1,54 \AA)$, operado com uma diferença de potencial no tubo de descarga de $40 \mathrm{kV}$ e uma corrente elétrica de $30 \mathrm{~mA}$. A varredura foi realizada na faixa de 5 a $50^{\circ}$ na velocidade de $2^{\circ} \cdot \mathrm{min}^{-1}$. Espectroscopia de absorção na região do infravermelho (FTIR): os espectros de absorção na região do infravermelho (IV) foram obtidos em um espectrômetro FTIR (Agilent, Cary 630), acoplado a um acessório ATR, na região de 4000 a $400 \mathrm{~cm}^{-1}$, e com $4 \mathrm{~cm}^{-1}$ de resolução. Ressonância magnética nuclear $(R M N)$ : os espectros de ressonância magnética nuclear do núcleo de ${ }^{29} \mathrm{Si}$ no estado sólido foram obtidos em um espectrômetro (Bruker, Avance III), operado a $400 \mathrm{MHz}$ na temperatura ambiente. A amostra sólida foi compactada em um rotor de óxido de zircônio de $7 \mathrm{~mm}$. As medidas foram obtidas em 59,61 $\mathrm{MHz}$ para o núcleo de ${ }^{29} \mathrm{Si}$, com rotação do ângulo mágico de $4 \mathrm{kHz}$. Análise termogravimétrica (ATG): as análises termogravimétricas foram obtidas em um instrumento (TA, $1090 \mathrm{~B}$ ), usando uma taxa de aquecimento de 0,167 K.s ${ }^{-1}$, sob um fluxo de argônio de $30 \mathrm{~cm}^{3} \cdot \mathrm{min}^{-1}$, na faixa entre a temperatura ambiente até aproximadamente $1000{ }^{\circ} \mathrm{C}$. Foram utilizadas massas iniciais dos sólidos em torno de $10 \mathrm{mg}$.

Experimentodeadsorção embatelada:opotencialde sorção de íons cobre pela montmorilonita organofuncionalizada, MMT-APTES, em solução aquosa, foi verificado pelo processo de batelada em flaconetes de polietileno de $10 \mathrm{~mL}$, contendo cerca de $25 \mathrm{mg}$ MMT-APTES e $3 \mathrm{~mL}$ de água deionizada. Foram utilizadas adições sucessivas de $20 \mu \mathrm{L}$ de uma solução estoque de cobre de concentração nominal de $0,1 \mathrm{~mol} \cdot \mathrm{dm}^{-3}$, preparada pela dissolução de nitrato de cobre hexa-hidratado $\left[\mathrm{Cu}\left(\mathrm{NO}_{3}\right)_{2} \cdot 6 \mathrm{H}_{2} \mathrm{O}\right]$ em água deionizada. A concentração correta dessa solução foi determinada por espectrometria de emissão óptica por plasma acoplado indutivamente (ICP-OES), utilizando uma solução padrão de cobre ICP/DCP 10,102 $\mu \mathrm{g} \cdot \mathrm{mL}^{-1}$. A técnica de batelada foi realizada em um banho agitador termostático (Marconi, MA-095). Após o procedimento de adsorção, os adsorventes foram separados das soluções aquosas por centrifugação a $4000 \mathrm{rpm}$ por $10 \mathrm{~min}$, retornando ao banho termostático por mais $1 \mathrm{~h}$, agora sem agitação. As concentrações finais de cobre remanescente nas soluções foram determinadas por ICP-OES em um espectrômetro (Perkin-Elmer, 3000 DV). Os sobrenadantes foram diluídos para adequar as concentrações à janela de leitura do equipamento. A quantidade de cobre adsorvido foi calculada pela aplicação da Eq. A [12]:

$$
\mathrm{N}_{\mathrm{f}}=\frac{\mathrm{C}_{\mathrm{o}}-\mathrm{C}_{\mathrm{e}}}{\mathrm{m}} \cdot \mathrm{V}
$$

em que $\mathrm{N}_{\mathrm{f}}$ é a quantidade de cobre adsorvido em mmol.g ${ }^{-1}$ de MMT-APTES, $\mathrm{C}_{0}$ e $\mathrm{C}_{\mathrm{e}}$ são as correspondentes concentrações inicial e de equilíbrio do íon, respectivamente, em mmol. $\mathrm{L}^{-1}$, $\mathrm{m}$ é a massa de MMT-APTES, em g, e $\mathrm{V}$ é o volume da solução em contato com o adsorvente, em L. O equilíbrio de adsorção foi estudado através dos modelos de Langmuir [13], Freundlich [14] e Sips [15], por ajuste não linear.
Titulação calorimétrica isotérmica (ITC): a titulação calorimétrica foi realizada num calorímetro isotérmico (Microcal, VP-ITC). Essencialmente uma massa de $15 \mathrm{mg}$ MMT-APTES foi suspensa em 1,43 mL de água deionizada, dentro da cela calorimétrica. Adições sucessivas de $5 \mu \mathrm{L}$ de solução de $\mathrm{Cu}^{2+}$ foram realizadas, registrando-se o respectivo efeito térmico, $\mathrm{q}_{\mathrm{T}}$, a cada adição. O procedimento foi repetido na ausência do sólido, para se obter o efeito térmico da diluição do titulante, $\mathrm{q}_{\mathrm{D}}$, a cada adição. Construíram-se duas curvas de $\Sigma \mathrm{q}_{\mathrm{T}}$ e de $\Sigma \mathrm{q}_{\mathrm{D}}$ em função do volume adicionado de titulante. A subtração gráfica, $\Sigma \mathrm{q}_{\mathrm{T}}-\Sigma \mathrm{q}_{\mathrm{D}}$, permitiu determinar a energia da adsorção, $\Sigma \mathrm{q}_{\mathrm{A}}$, a cada volume adicionado de solução de $\mathrm{Cu}^{2+}$. A variação entalpia na adsorção, ao longo da titulação, pôde ser calculada pela relação:

$$
\Delta \operatorname{adsH}=\frac{\Sigma_{\mathrm{qA}}}{\mathrm{N}_{\mathrm{f}}}
$$

\section{RESULTADOS E DISCUSSÃO}

Difratometria de raios $X(D R X)$ : o difratograma da montmorilonita (MMT-Na) está apresentado na Fig. 1a. Nela se observam as reflexões dos planos (001), (100), (110), (210) e (060), características da montmorilonita, situadas em $6,12^{\circ}, 19,95^{\circ}, 35,10^{\circ}, 54,04^{\circ}$ e $62,02^{\circ}$, respectivamente [16-19]. O primeiro plano (001) está associado ao plano cristalográfico principal da estrutura do argilomineral, enquanto o último (060) é considerado, segundo a literatura, a impressão digital da montmorilonita, pois discrimina as espécies di ou trioctaédricas, que neste caso se trata de uma montmorilonita dioctaédrica [20]. O difratograma da montmorilonita intercalada com o surfactante CTAB (MMT-CTAB) encontra-se na Fig. 1b e nele se observa um aumento do espaçamento basal $\mathrm{d}_{001}$ de 14,43 para $18,47 \AA$. Isso indicou que o surfactante foi intercalado no espaço interlamelar da matriz inorgânica [21, 22]. O difratograma da amostra organofuncionalizada MMT-CTAB-APTES (Fig. 1c) não apresentou nenhum aumento no espaçamento basal, apenas uma diminuição da intensidade dos picos, quando comparado com o difratograma da amostra MMT-CTAB (Fig. 1b); isso indicou que o organossilano influenciou na cristalinidade e no tamanho do cristal da amostra. Após a remoção do CTAB, o pico do plano (001) da montmorilonita MMT-APTES retornou para posição da montmorilonita inicial, o que implica que imobilização das moléculas do organossilano não ocorreu na região interlamelar (Fig. 1d).

Espectroscopia de absorção na região do infravermelho (FTIR): o espectro de infravermelho da montmorilonita (MMT-Na) está disposto na Fig. 2a. Nele se observa uma banda de absorção em $3627 \mathrm{~cm}^{-1}$ correspondente ao estiramento $v(\mathrm{O}-\mathrm{H})$ de hidroxilas estruturais localizadas entre as camadas octaédricas e tetraédricas da amostra. As bandas em 3409 e $1636 \mathrm{~cm}^{-1}$ se referem ao estiramento $v(\mathrm{O}-\mathrm{H})$ e a deformação $\delta(\mathrm{O}-\mathrm{H})$ das moléculas de água adsorvidas e presentes no espaçamento interlamelar. As bandas em 1110 e 1000 $\mathrm{cm}^{-1}$ foram atribuídas ao estiramento do grupo $v(\mathrm{Si}-\mathrm{O}-\mathrm{Si})$. Observaram-se bandas de deformação $\delta(\mathrm{O}-\mathrm{H})$ em torno de 


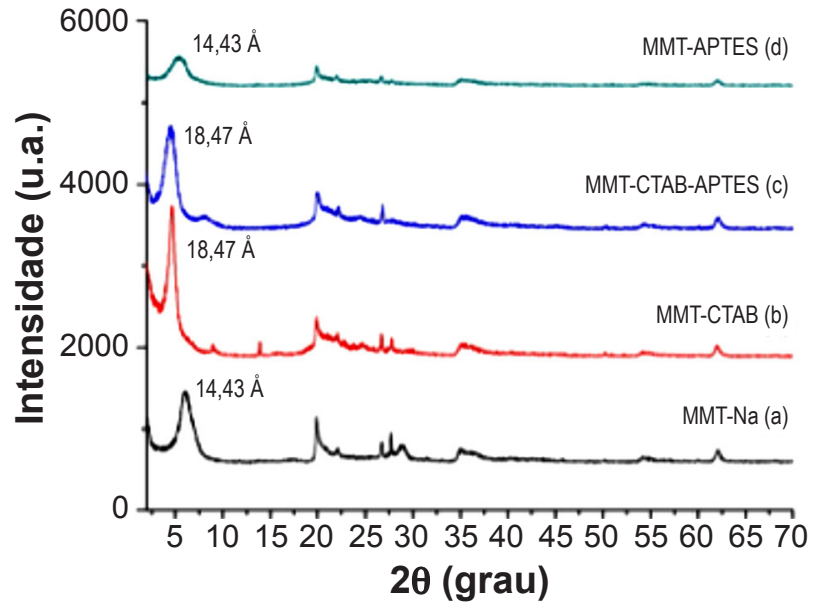

Figura 1: Difratogramas de raios X das amostras MMT-Na (a), MMT-CTAB (b), MMT-CTAB-APTES (c) e MMT-APTES (d). [Figure 1: X-ray diffraction patterns of the samples MMT-Na (a), $\operatorname{MMT-CTAB}(b), M M T-C T A B-A P T E S(c)$, and MMT-APTES (d).]

920 e $790 \mathrm{~cm}^{-1}$ atribuídas aos grupos (AlAlOH) e (AlMgOH), respectivamente. A banda em $528 \mathrm{~cm}^{-1}$ corresponde às ligações de deformação $\delta(\mathrm{Al}-\mathrm{O}-\mathrm{Si})$, sendo o silício ligado ao sítio tetraédrico e o alumínio ao sítio octaédrico do argilomineral [18, 23-29]. Após a organofuncionalização a amostra MMT-APTES (Fig. 2b) apresentou novas bandas em torno de 2926 e $2852 \mathrm{~cm}^{-1}$ atribuídas ao estiramento simétrico e assimétrico do grupo $v\left(\mathrm{CH}_{2}\right)$, respectivamente, indicando a presença do organossilano na estrutura da mesma [23,30-33]. Outras bandas características da presença do organossilano foram observadas em 1472 e $626 \mathrm{~cm}^{-1}$ correspondentes às deformações simétricas do grupo $\delta_{\mathrm{s}}\left(\mathrm{NH}_{2}\right)$ e $\delta_{\mathrm{s}}\left(\mathrm{CH}_{2}\right)$. Estes resultados corroboraram com os observados na literatura [26, 30, 31, 34-36].

Análise termogravimétrica (ATG): as curvas termogravimétricas das amostras MMT-Na, MMT-CTAB, MMT-CTAB-APTES e MMT-APTES estão apresentadas na Fig. 3. Na curva da amostra MMT-Na observam-se duas etapas principais de perda de massa. A primeira etapa

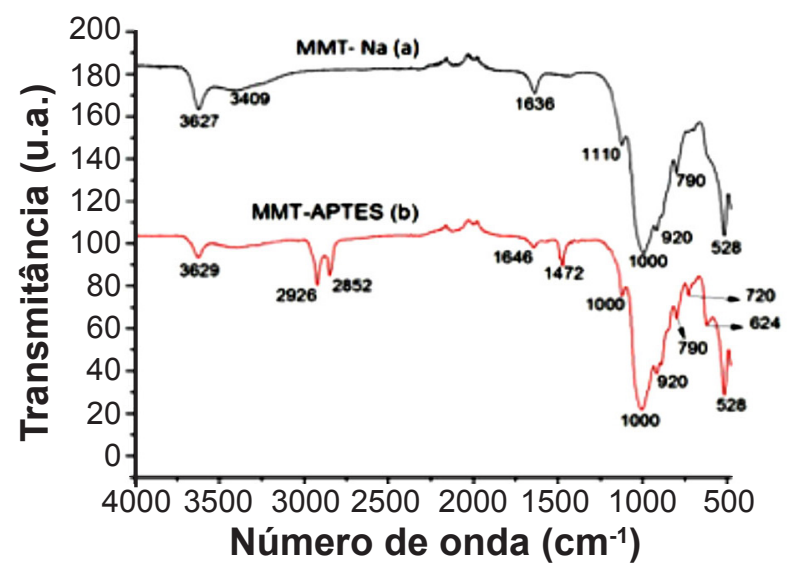

Figura 2: Espectros de infravermelho das amostras MMT-Na (a) e MMT-APTES (b).

[Figure 2: Infrared spectra of samples MMT-Na (a) and MMTAPTES (b).] ocorreu a partir da temperatura ambiente até $100{ }^{\circ} \mathrm{C}(9 \%)$, sendo atribuída à dessorção de água adsorvida nos poros e na superfície da argila. A segunda perda ocorreu na faixa de 580 a $720{ }^{\circ} \mathrm{C}$ atribuída a desidroxilação de grupos $\mathrm{OH}$ estruturais, que correspondeu a uma perda de $3 \%$ de massa. Segundo a literatura, esses resultados sugerem que a MMT$\mathrm{Na}$ é relativamente estável e que sua estrutura se mantém, mesmo em temperaturas elevadas [6, 37-39]. Na curva termogravimétrica da amostra intercalada com o surfactante (MMT-CTAB) observou-se uma pequena perda de massa a partir da temperatura ambiente até $140{ }^{\circ} \mathrm{C}$, que correspondeu à água adsorvida (menor que $1 \%$ ). As perdas ocorridas na faixa entre 180 e $420{ }^{\circ} \mathrm{C}$ foram atribuídas à degradação térmica da estrutura do CTA (23\%), sendo a 'cauda alquila' $-\mathrm{CH}_{2}$ e a 'cabeça amônio' - $\mathrm{N}\left(\mathrm{CH}_{3}\right)_{3}$. A perda de massa na faixa de 450 a $600{ }^{\circ} \mathrm{C}$ foi relacionada à decomposição do restante da cadeia alquila (1\%). A perda de massa a partir de $600{ }^{\circ} \mathrm{C}$ foi relacionada à eliminação da água da rede cristalina e decomposição de resíduos da cadeia carbônica (2\%). Esses resultados indicaram a presença do CTA no espaço interlamelar da montmorilonita [40-43].

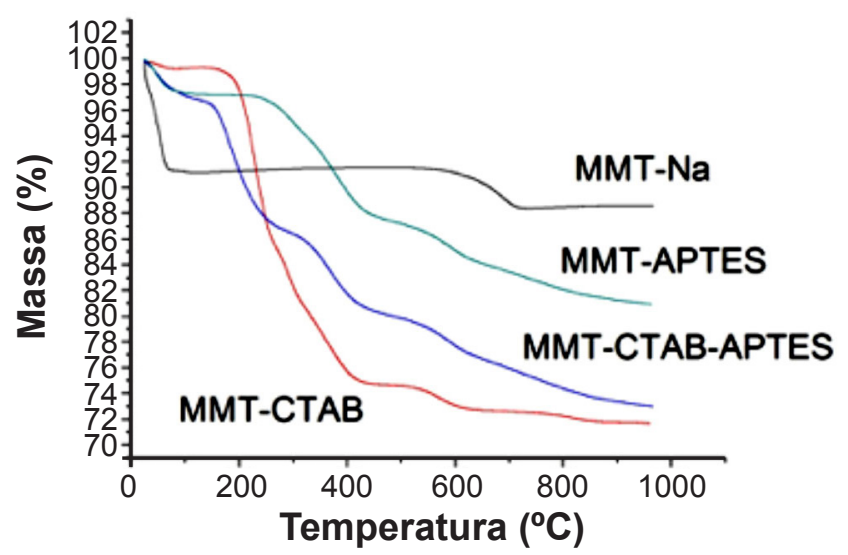

Figura 3: Curvas termogravimétricas das amostras MMT-Na, MMT-CTAB, MMT-CTAB-APTES E MMT-APTES.

[Figure 3: Thermogravimetric curves of the samples MMT-Na, MMT-CTAB, MMT-CTAB-APTES and MMT-APTES.]

$\mathrm{Na}$ curva termogravimétrica da amostra organofuncionalizada (MMT-CTAB-APTES) a perda de massa entre a temperatura ambiente e $150{ }^{\circ} \mathrm{C}$ foi atribuída à dessorção de água fisicamente adsorvida (4\%). Houve quatro perdas de massas que podem ser interpretadas como degradações do CTA e do organossilano presentes. A degradação térmica da parte orgânica dessas moléculas deve ter ocorrido concomitantemente, por isso a interpretação foi dificultada, e isso ocorreu no intervalo de 200 a $750^{\circ} \mathrm{C}$. Para o CTA vale a interpretação anterior para o sólido MMT-CTAB. Para o APTES presente, a primeira degradação, entre 200 e $350^{\circ} \mathrm{C}$, foi atribuída à remoção do silano funcionalizado nas bordas e/ou adsorvido na superfície da montmorilonita. A perda de massa entre 350 e $450{ }^{\circ} \mathrm{C}$ se refere à remoção do silano do espaço interlamelar. A perda entre 450 e $600{ }^{\circ} \mathrm{C}$ 
deve-se à degradação do silano funcionalizado nas camadas e no espaço interlamelar. A perda de massa entre 600 e 750 ${ }^{\circ} \mathrm{C}$ foi atribuída à degradação das camadas de silicato de alumínio [23, 32, 33, 36, 44, 45]. É importante comentar que embora seja possível calcular as porcentagens de perda em cada faixa comentada, como se observa nos dados da Tabela I, não se pôde atribuir esses valores especificamente a uma degradação, devido à presença de dois tipos de moléculas. Na curva termogravimétrica da amostra MMT-APTES, a perda de massa que ocorreu entre a temperatura ambiente e $107^{\circ} \mathrm{C}$ foi atribuída à água adsorvida fisicamente. A perda na faixa entre 251 e $600^{\circ} \mathrm{C}$ correspondeu à decomposição do silano quimicamente ligado à estrutura do silicato lamelar [23, 45, 46]. Valem, aqui, as discussões apresentadas anteriormente, relativas ao APTES. As porcentagens de perdas e intervalos encontram-se na Tabela I. Nesta tabela estão listados os percentuais de perda de massa e os respectivos intervalos de temperatura para cada estágio das degradações térmicas. Esses resultados foram obtidos utilizando-se as curvas derivadas (DTG).

Ressonância magnética nuclear: o espectro de ressonância do núcleo de silício da MMT-Na (Fig. 4a) apresenta dois picos. O primeiro, em aproximadamente -92 ppm $\left(\mathrm{Q}^{3}\right)$, foi atribuído ao átomo de Si central em (Si$\mathrm{O}-)_{2} \mathrm{Si}(-\mathrm{O}-\mathrm{Al})-\mathrm{OH}$, e o segundo pico por volta de $-107 \mathrm{ppm}$ $\left(\mathrm{Q}^{4}\right)$ se deve ao átomo central de $\mathrm{Si}$ em (Si-O-) $)_{3} \mathrm{Si}(-\mathrm{O}-\mathrm{Al})$ [47]. Na Fig. 4b, que mostra o espectro a amostra MMTCTAB, observa-se que não houve nenhuma alteração após a intercalação com o CTAB; assim é possível inferir que não existiu nenhuma modificação estrutural causada pelo surfactante. Como pode ser observado na Fig. 4c, no espectro de ${ }^{29} \mathrm{Si}$ da amostra MMT-CTAB-APTES, surge um pico largo próximo de -68 ppm $\left(\mathrm{T}^{3}\right)$. Este novo pico se deve à fixação das

Tabela I - Intervalo de temperatura $(\Delta \mathrm{T})$ e porcentagem de perda de massa $(\Delta \mathrm{m})$ dos materiais estudados.

[Table I - Temperature range $(\Delta T)$ and percentage of mass loss $(\Delta m)$ of the studied materials.]

\begin{tabular}{ccc}
\hline Material & $\Delta \mathrm{T}\left({ }^{\circ} \mathrm{C}\right)$ & $\Delta \mathrm{m}(\%)$ \\
\hline \multirow{2}{*}{ MMT-Na } & $25-100$ & 9 \\
& $580-720$ & 3 \\
\hline & $25-140$ & 0,8 \\
MMT- & $180-420$ & 23 \\
CTAB & $450-600$ & 1 \\
& $600-880$ & 2 \\
\hline & $25-150$ & 4 \\
MMT- & $200-350$ & 11 \\
CTAB- & $350-450$ & 7 \\
APTES & $450-600$ & 3 \\
& $600-750$ & 4 \\
\hline MMT- & $25-107$ & 3 \\
APTES & $251-600$ & 13 \\
\hline
\end{tabular}

espécies de silano na superfície da montmorilonita, através da ligação covalente entre os átomos de silício e carbono $\left[\mathrm{Si}(\mathrm{OSi})_{3} \mathrm{R}\right]$ [48]. O espectro da amostra MMT-APTES (Fig. 4d) apresenta o pico $\left(\mathrm{T}^{3}\right)$, o que sugere a conservação da ligação entre as espécies do silano e a matriz inorgânica, mesmo após o procedimento de remoção do cátion $\mathrm{CTA}^{+}$.

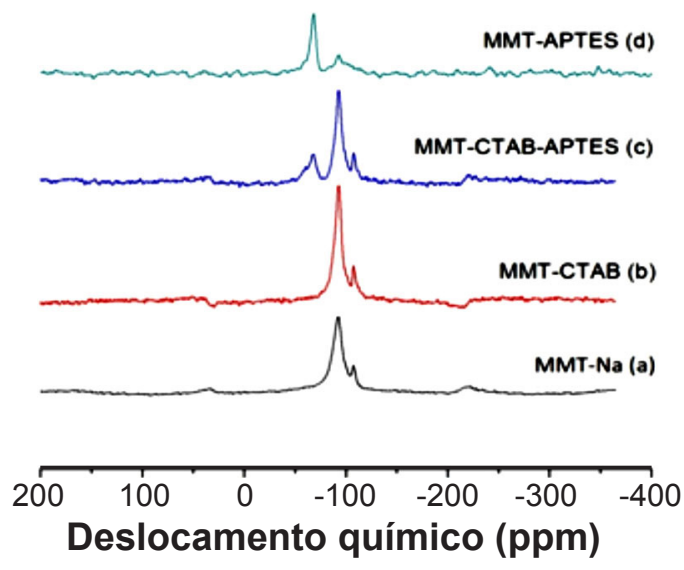

Figura 4: Espectro de RMN ${ }^{29} \mathrm{Si}$ das amostras MMT-Na (a), MMTCTAB (b), MMT-CTAB-APTES (c) e MMT-APTES (d).

[Figure 4: ${ }^{29} \mathrm{Si}$ NMR spectra of the samples MMT-Na (a), MMTCTAB (b), MMT-CTAB-APTES (c), and MMT-APTES (d).]

Análise elementar: um dos objetivos da análise elementar é determinar o grau de organofuncionalização do material sintetizado. A partir da análise de $\mathrm{C}, \mathrm{H}$ e $\mathrm{N}$ calculou-se a quantidade em mol de nitrogênio em $1 \mathrm{~g}$ do material e, considerando-se as proporções atômicas na molécula do agente sililante, calculou-se o grau de funcionalização, ou a densidade de grupos imobilizados (@). Os dados de análise elementar também servem para fazer considerações sobre a presença de moléculas orgânicas no sólido, sobre relações entre quantidades presentes quando há mais de um tipo de molécula, assim como é possível fazer comentários correlacionando-se esses dados aos dados de ATG e ressonância, tudo relativo à questão de 'quantidades'. Os dados da análise elementar encontram-se na Tabela II. A densidade de grupos imobilizados para MMT-APTES foi 1,46 mmol.g $\mathrm{g}^{-1}$. Observa-se, ainda, que os teores de $\mathrm{C}$ e $\mathrm{N}$ diminuíram em MMT-APTES em comparação com MMTCTAB-APTES, o que sugeriu a remoção dos cátions $\mathrm{CTA}^{+}$ da estrutura da matriz inorgânica.

Embora as incertezas nas determinações de perdas de massa registradas pela ATG sejam bem maiores do que as incertezas da análise elementar, sendo na ATG em torno de 3\%, uma comparação dos resultados entre as Tabelas I e II mostra uma excelente concordância entre os dados. Para a MMT-CTAB, a perda de massa relativa à matéria orgânica entre 180 e $600{ }^{\circ} \mathrm{C}$ foi de $\sim 27 \%$, o mesmo valor encontrado pela análise elementar somando-se $\mathrm{C}, \mathrm{H}$ e N. Para os outros materiais, essa comparação deve ser feita desconsiderandose o hidrogênio, já que essas amostras apresentaram perdas de massa entre a temperatura ambiente e $200{ }^{\circ} \mathrm{C}$, o que 
Tabela II - Percentuais de C, H e $\mathrm{N}$ obtidos através análise elementar e as densidades de grupos imobilizados (Q) por grama de material.

[Table II - Percentages of $C, H$ and $N$ obtained by elemental analysis and the densities of immobilized groups $(\mathrm{Q})$ per gram of material.]

\begin{tabular}{ccccccc}
\hline Composto & $\mathrm{C}(\%)$ & $\mathrm{H}(\%)$ & $\mathrm{N}(\%)$ & $\varrho\left(\mathrm{mmol} . \mathrm{g}^{-1}\right)$ & Grupo & $\mathrm{C} / \mathrm{N}$ no material \\
\hline MMT-Na & 0,31 & 0,98 & 0,07 & - & - & - \\
MMT-CTAB & 21,35 & 4,42 & 1,36 & - & $\mathrm{C}_{19} \mathrm{H}_{42} \mathrm{~N}^{+}$ & $18: 1$ \\
MMT-CTAB-APTES & 18,00 & 3,96 & 2,32 & - & - & $9: 1$ \\
MMT-APTES & 10,95 & 2,61 & 2,05 & 1,46 & $\mathrm{C}_{9} \mathrm{H}_{23} \mathrm{NO}_{3} \mathrm{Si}$ & $6: 1$ \\
\hline
\end{tabular}

significa que ainda poderia haver água adsorvida quando da análise elementar. Assim, levando-se em conta apenas $\mathrm{C}$ e $\mathrm{N}$ na análise elementar, para a MMT-CTAB-APTES observaram-se $21 \%$ e $20,3 \%$ de matéria orgânica pela ATG e análise elementar, respectivamente. Para MMT-APTES os resultados foram iguais a $13 \%$. Por outro lado, observando-se as relações $\mathrm{C}: \mathrm{N}$, observou-se que para o caso do MMT-CTAB a proporção molar foi de 18:1, valor muito próximo de 19:1 esperado pela fórmula química do CTAB. Já quando se tem MMT-CTAB-APTES essa relação passa para 9:1, o esperado para a molécula do APTES. Isso poderia ser interpretado como uma ausência do CTAB na amostra, no entanto, quando se observa a relação C:N para o MMT-APTES, esse valor cai para 6:1, valor abaixo do esperado para o APTES livre. No entanto, quando se observa o espectro de RMN, vêse que o silano ao se ligar à matriz sólida o faz de diferentes formas, mas sempre implicando na remoção de parte de sua molécula, a que contém carbono e não contém nitrogênio, o que justificaria a diminuição na relação $\mathrm{C}: \mathrm{N}$.

Isotermas de adsorção: a distribuição de íons metálicos entre as fases líquida e sólida é geralmente descrita por modelos de adsorção como os de Langmuir, Freundlich, Sips, entre outros. Em essência, todos os modelos de isotermas procuram equacionar a quantidade de material adsorvido em função de alguma variável de equilíbrio, como a pressão ou a concentração. Em geral, os modelos se aplicam muito bem a sistemas gás-sólido, mas apresentam dificuldades na sua aplicação a sistemas condensados (líquido-sólido), já que o solvente compete com o adsorvato pelos sítios de adsorção. O modelo de Langmuir, originalmente desenvolvido para estudos de adsorção de gases em sólidos, baseia-se na suposição de que o adsorvato é quimicamente adsorvido por uma quantidade fixa (máxima) de sítios $\left(\mathrm{N}_{\mathrm{s}}\right)$, que cada sítio da superfície só interage com uma molécula do adsorvato (1:1), que todos os sítios são energeticamente equivalentes e que não há interação entre as moléculas do adsorvato na superfície do sólido [13]. A equação matemática que descreve esse modelo é:

$$
\mathrm{N}_{\mathrm{f}}=\frac{\mathrm{N}_{\mathrm{S}} \cdot \mathrm{K}_{\mathrm{L}} \cdot \mathrm{C}_{\mathrm{e}}}{1+\mathrm{K}_{\mathrm{L}} \cdot \mathrm{C}_{\mathrm{e}}}
$$

em que $\mathrm{N}_{\mathrm{f}}$ é a quantidade do adsorvato adsorvido no equilíbrio, $\mathrm{N}_{\mathrm{s}}$ é a capacidade máxima de adsorção relacionada à cobertura de uma monocamada, $\mathrm{K}_{\mathrm{L}}$ é a constante de afinidade de Langmuir e $\mathrm{C}_{\mathrm{e}}$ é a concentração do adsorvato na fase aquosa no equilíbrio. O modelo de Freundlich assume que a concentração do adsorvato na superfície do adsorvente aumenta infinitamente com a concentração do adsorvato [14]. Esse modelo é amplamente aplicado a sistemas heterogêneos [49]. A equação matemática que descreve esse modelo é:

$$
\mathrm{N}_{\mathrm{f}}=\mathrm{K}_{\mathrm{F}} \cdot \mathrm{C}_{\mathrm{e}}^{\frac{1}{n}}
$$

em que $K_{F}$ e $n$ são as constantes de Freundlich, que apontam para a capacidade relativa e intensidade de adsorção, respectivamente. Ambos parâmetros são dependentes da temperatura. O modelo de Sips, também conhecido como Langmuir-Freundlich, como o nome indica, é uma combinação dos modelos de Langmuir e Freundlich [15]. A equação matemática que descreve o modelo de Sips é descrita pela Eq. E:

$$
\mathrm{N}_{\mathrm{f}}=\frac{\mathrm{N}_{\mathrm{S}} \cdot \mathrm{K}_{\mathrm{S}} \cdot \mathrm{C}_{\mathrm{e}}^{\frac{1}{n}}}{1+\mathrm{K}_{\mathrm{S}} \cdot \mathrm{C}_{\mathrm{e}}^{\frac{1}{n}}}
$$

Tabela III - Parâmetros das isotermas para adsorção de $\mathrm{Cu}^{2+}$. Condições: $25 \pm 1{ }^{\circ} \mathrm{C}, 25$ mg de amostra, $\mathrm{pH} 6,5$, tempo de contato $8 \mathrm{~h}$.

[Table III - Isotherm parameters for adsorption of $\mathrm{Cu}^{2+}$. Conditions: $25 \pm 1{ }^{\circ} \mathrm{C}, 25 \mathrm{mg}$ sample, $p H 6.5$, contact time 8 h.]

\begin{tabular}{ccccccccccc}
\hline \multicolumn{3}{c}{ Langmuir } & \multicolumn{3}{c}{ Freundlich } & \multicolumn{4}{c}{ Sips } \\
$\mathrm{N}_{\mathrm{s}}$ & $\mathrm{K}_{\mathrm{L}}$ & $\mathrm{r}^{2}$ & $\mathrm{~K}_{\mathrm{F}}$ & $\mathrm{n}_{\mathrm{F}}$ & $\mathrm{r}^{2}$ & $\mathrm{~N}_{\mathrm{s}}$ & $\mathrm{K}_{\mathrm{s}}$ & $\mathrm{n}_{\mathrm{s}}$ & $\mathrm{r}^{2}$ \\
\hline 0,41 & 273 & 0,948 & 3,89 & 1,88 & 0,983 & 2,59 & 2,08 & 0,57 & 0,981 \\
\hline
\end{tabular}


em que $\mathrm{K}_{\mathrm{s}}$ é a constante de Sips relacionada à constante de afinidade e $\mathrm{N}_{\mathrm{s}}$ é capacidade máxima de adsorção para o modelo. Em baixas concentrações, a equação de Sips se reduz à de Freundlich, enquanto que em altas concentrações, quando se aproxima a formação de monocamada, a equação se aproxima à de Langmuir.

No caso aqui específico, os parâmetros das isotermas foram determinados por regressão não linear e os coeficientes de determinação encontrados evidenciaram que os dados não se comportaram bem conforme os modelos (Tabela III). Os modelos preveem uma capacidade máxima de adsorção, mas esse valor não foi atingido na faixa experimental utilizada; as curvas de $\mathrm{N}_{\mathrm{f}}$ em função da concentração não atingiram um patamar, o que prejudicou uma determinação mais exata e precisa da capacidade máxima de adsorção $\left(\mathrm{N}_{\mathrm{s}}\right)$. Observou-se que os valores de $\mathrm{N}_{\mathrm{s}}$ encontraram-se muito aquém do valor esperado pela análise de nitrogênio, que mostrou uma funcionalização de $1,46.10^{-3} \mathrm{~mol}$ de nitrogênio (sítios potenciais) de adsorção por grama da amostra. A diferença entre essas grandezas, $\mathrm{N}_{\mathrm{s}}$ e densidade de funcionalização, pode se originar de fontes diversas, inclusive da dificuldade em se obter dados 'seguros' de quantidades para determinadas faixas de concentração. Em baixos valores de concentração a adsorção é elevada e há dificuldades na determinação da concentração de equilíbrio e para valores altos de concentração, embora haja maior adsorção, a concentração de equilíbrio, percentualmente, está muito próxima da concentração inicial. A dificuldade então advém, pois as quantidades adsorvidas são obtidas por diferenças entre as duas concentrações, inicial e de equilíbrio. Do ponto de vista estatístico, analisando somente as curvas obtidas (Fig. 5), pelo fato de a porcentagem de sítios ocupados ter sido baixa na faixa de concentração estudada, as extrapolações e os ajustes, para qualquer modelo, ficaram prejudicados, obtendo-se altos valores de

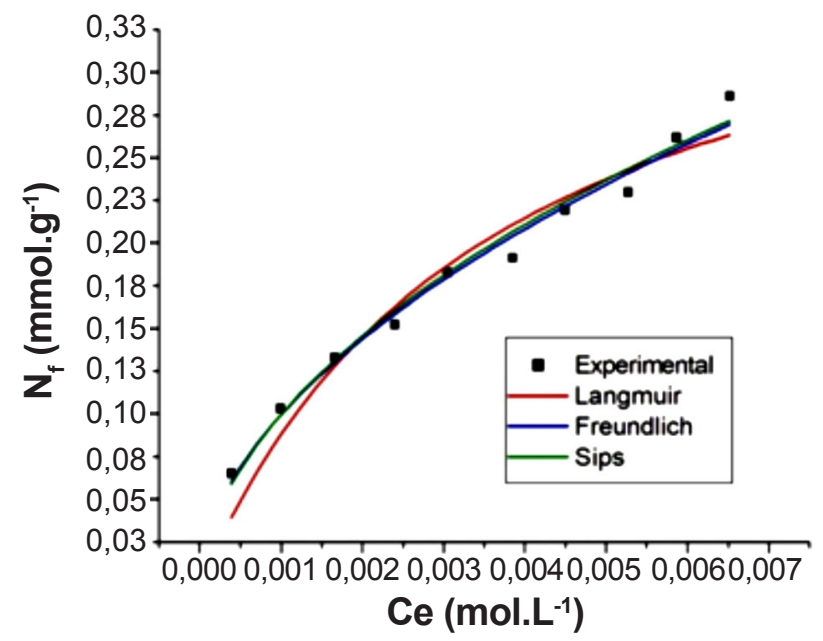

Figura 5: Modelos de isotermas para remoção de $\mathrm{Cu}^{2+}$ em solução aquosa pela amostra MMT-APTES. Condições: $25 \pm 1^{\circ} \mathrm{C}, 25 \mathrm{mg}$ de amostra, $\mathrm{pH} 6,5$, tempo de contato $8 \mathrm{~h}$.

[Figure 5: Isotherm models for removal of $\mathrm{Cu}^{2+}$ in aqueous solution by the MMT-APTES sample. Conditions: $25 \pm 1{ }^{\circ} \mathrm{C}, 25 \mathrm{mg}$ of sample, $\mathrm{pH} 6.5$, contact time $8 \mathrm{~h}$.]
Tabela IV - Capacidades máximas de adsorção de diferentes adsorventes para remoção de cobre de meios aquosos.

[Table IV - Maximum adsorption capacities of different adsorbents for removal of copper from aqueous media.]

\begin{tabular}{ccc}
\hline Adsorvente & $\mathrm{N}_{\mathrm{s}}\left(\mathrm{mg} \cdot \mathrm{g}^{-1}\right)$ & Referência \\
\hline Casca de trigo & 17,4 & {$[50]$} \\
Ca-montmorilonita & 14,8 & {$[51]$} \\
Nanopartículas de $\gamma$-alumina & 14,5 & {$[52]$} \\
Na-montmorilonita & 3,04 & {$[53]$} \\
MMT-APTES & 26,1 & Este trabalho \\
\hline
\end{tabular}

Nota: valores de $N_{s}$ obtidos pelo ajuste do modelo Langmuir.

incertezas nos parâmetros obtidos nas regressões. Essas são dificuldades esperadas em estudos de isotermas de adsorção em meio condensado, no entanto raramente são comentadas na literatura.

Como é discutido mais adiante, as isotermas de adsorção foram estudadas em três diferentes temperaturas: 25, 35 e 45 ${ }^{\circ} \mathrm{C}$. No entanto, o tratamento dos dados levou a resultados parecidos em relação às incertezas nos três casos, por isso são discutidos, mais apropriadamente, em conjunto com os resultados de calorimetria. Em vista das dificuldades anteriores, e considerando-se o fato de que os resultados do estudo das isotermas seriam utilizados em conjunto com os resultados da calorimetria, optou-se por uma metodologia distinta no tratamento dos dados obtidos. No entanto, como comparação, a Tabela IV mostra as capacidades máximas de adsorção $\left(\mathrm{N}_{\mathrm{s}}\right)$ relatadas na literatura para diferentes tipos de adsorventes utilizados na adsorção de cobre a partir de soluções aquosas. $\mathrm{O}$ valor de $\mathrm{N}_{\mathrm{s}}$ neste trabalho foi obtido pelo ajuste do modelo Langmuir. Como se observa nessa tabela, o material estudado neste trabalho possuiu capacidade de adsorção de $\mathrm{Cu}^{2+}$ comparável com alguns outros materiais e até maior em alguns casos.

Termodinâmica de adsorção: a termodinâmica é uma ferramenta largamente utilizada no estudo do comportamento da adsorção. Os parâmetros, incluindo a variação da energia livre de Gibbs $(\Delta G)$, a variação de entalpia $(\Delta H)$ e variação da entropia $(\Delta S)$, têm sido obtidos ao longo do tempo nos mais diversos estudos de adsorção. Um tratamento matemático bastante comum nesses casos é usar os dados das isotermas, aplicando-se diversos modelos, e obter algum parâmetro (constante) dessa modelagem que tenha alguma correlação com uma suposta 'constante termodinâmica de equilíbrio'. A partir daí, as Eqs. F e G são utilizadas na obtenção das supostas grandezas termodinâmicas, $\Delta \mathrm{G}, \Delta \mathrm{H}$ e $\Delta S$ :

$$
\begin{aligned}
& \Delta \mathrm{G}=-\mathrm{R} T \ln \mathrm{K} \\
& \ln \mathrm{K}=\frac{-\Delta \mathrm{H}}{\mathrm{R}} \frac{1}{\mathrm{~T}}+\frac{\Delta \mathrm{S}}{\mathrm{R}}
\end{aligned}
$$

Assim, a constante de equilíbrio e a variação da energia livre de Gibbs são grandezas semelhantes, porém em diferentes escalas. Em alguns campos da química 
interessa expressar o equilíbrio em termos de quantidades, aparecendo valores numéricos de constantes, como as constantes de solubilidade, constantes de ionização, etc.; em outros campos o equilíbrio se expressa na escala de energia, mais propriamente, energia livre de Gibbs. As escalas de $\mathrm{K}$ e $\Delta \mathrm{G}$, são interconvertidas pela expressão dada pela Eq. F. Os valores de $\mathrm{K}$ são obtidos direta e experimentalmente por medidas de quantidades (massa, concentração, pressão, etc.), enquanto que os de $\Delta \mathrm{G}$ não são obtidos diretamente.

Embora de grande utilidade prática, por vezes, os aspectos mais fenomenológicos de um dado processo químico são mais bem entendidos observando-se de que forma a variação de entalpia e de entropia contribuem para o valor de $\Delta \mathrm{G}$. A Eq. $\mathrm{G}$ mostra a relação matemática entre esses três parâmetros termodinâmicos, $\Delta \mathrm{G}, \Delta \mathrm{H}$ e $\Delta \mathrm{S}$, a uma dada temperatura. A forma direta de se obter esses parâmetros num dado processo consiste, então, em se determinar os valores da constante $\mathrm{K}$, por medidas de quantidades, e da variação de entalpia, $\Delta \mathrm{H}$, em um calorímetro, por exemplo, e calcular $\Delta \mathrm{G}$ e $\Delta \mathrm{S}$, aplicando-se as Eqs. F e G. Por outro lado, quando não se pode medir $\Delta \mathrm{H}$ diretamente em um calorímetro, determinamse valores de constante de equilíbrio a diferentes temperaturas e aplica-se a relação de Van't Hoff a esses valores, ou seja, utiliza-se a Eq. G, correlacionando-se $\operatorname{lnK}$ e $1 / T$, obtendo-se os valores de $\Delta \mathrm{H}$ e $\Delta \mathrm{S}$. Esse tratamento matemático, no entanto, pressupõe uma verdadeira constante de equilíbrio. No caso da adsorção, entretanto, ao longo do tempo, pela dificuldade em se estabelecer referências adequadas à adsorção, tornou-se muito comum na literatura fazer-se uma 'equivalência' entre as constantes de várias modelagens matemáticas (modelos de isoterma de adsorção) com uma 'verdadeira' constante de equilíbrio. Assim, de acordo com essa metodologia de cálculo, isotermas a várias temperaturas são obtidas e os valores de 'constantes' para algum dos modelos de isoterma são obtidos e então se aplicam as Eqs. F e G a essas 'constantes' (métodos da segunda lei da termodinâmica).

Vale à pena observar que o modelo que mais se aproxima de uma definição de expressão de constante de equilíbrio convencional (IUPAC) é o modelo de Langmuir. Mesmo nesse caso, no entanto, a grande dificuldade é estabelecer uma concentração de referência para a adsorção. Como não há um critério definido para essa concentração de referência (seria $1 \mathrm{~g} . \mathrm{dm}^{-3}, 1 \mathrm{~mol} . \mathrm{dm}^{-3}, 1 \mathrm{mg} \cdot \mathrm{dm}^{-3}$ ?), os valores de constantes obtidos por aplicação desse modelo acabam sendo contaminados pela unidade de concentração utilizada. Também vale a pena destacar que esses modelos não foram pensados para se determinar uma constante de equilíbrio verdadeira; eles foram pensados para se determinar uma equação matemática que melhor expresse a dependência da quantidade de adsorvato adsorvido em função de sua concentração em equilíbrio na solução. A partir dessa modelagem, e por falta de uma referência de concentração, na literatura passou-se a adotar essas constantes como sendo constantes de equilíbrio e, como consequência disso, nem sequer um consenso sobre a unidade de concentração a ser utilizada está estabelecido. Isso, muitas vezes, leva a valores de 'parâmetros termodinâmicos' impossíveis de se imaginar, sendo essa a maior dificuldade que se interpõe nesse tipo de tratamento. Para uma leitura bem detalhada desse problema, pode-se consultar a literatura [54].

Outro ponto a destacar é o fato de que cada sítio de adsorção pode ter características próprias e, portanto, diferentes propriedades adsortivas; raramente se tem uma superfície de sítios homogêneos. Como o próprio valor de $\mathrm{K}$ nessa metodologia é obtido por linearização dos dados da isoterma, isso leva a crer que tanto $\Delta \mathrm{G}$ como $\Delta \mathrm{H}$ e $\Delta \mathrm{S}$ não são exatamente as verdadeiras funções termodinâmicas no processo de adsorção; seriam valores médios. Também vale ressaltar que cada modelo matemático de adsorção pode levar a diferentes valores de constante de afinidade e, por isso mesmo, a diferentes valores de $\Delta \mathrm{G}, \Delta \mathrm{H}$ e $\Delta \mathrm{S}$. No caso da adsorção, o que geralmente se faz é utilizar o modelo matemático que melhor se adequa aos dados experimentais e, dessa forma, proceder ao cálculo das grandezas termodinâmicas e sua discussão. Isso, no entanto, não significa que está tudo certo. Como descrito anteriormente, a modelagem de Langmuir é a que mais se aproxima de um tratamento matemático esperado para o equilíbrio, já que o modelo se baseia em sítios homogêneos, com interações 1 para 1 entre adsorvato e adsorvente e também que se forma apenas uma monocamada. A única dificuldade é, então, estabelecer um referencial para a concentração, no caso da constante de equilíbrio, ou um referencial de potencial químico para o soluto em solução, no caso de $\Delta \mathrm{G}$.

No caso aqui presente, inicialmente procurou-se determinar a variação de entalpia na adsorção pela técnica da titulação calorimétrica, como descrito anteriormente. Para isso foi utilizado um calorímetro (Thermometric, 2277). Apareceram dois pequenos sinais no início da titulação (adição de solução de $\mathrm{Cu}^{2+}$ ) e depois o que se observou foram sinais muito parecidos aos da diluição dessa solução. Por conta disso, optou-se por realizar a titulação calorimétrica em um sistema calorimétrico mais sensível, o microcalorímetro (Microcal, VP-ITC), o que permitiu a obtenção dos dados. Também nesse aparelho os sinais de energia foram muito pequenos, mas observáveis em todos os pontos da titulação. Adicionalmente, resolveu-se utilizar o método da segunda lei, como descrito anteriormente, até como forma de verificar se as possíveis constantes da modelagem das isotermas poderiam refletir esse pequeno valor de entalpia determinada via titulação calorimétrica. Pelo fato de que os dados não haviam se ajustado bem aos modelos, foi realizado um tratamento dos dados, tomando por base o modelo de Langmuir. Inicialmente considerou-se a capacidade máxima de adsorção, $\mathrm{N}_{\mathrm{s}}$, como sendo igual à densidade de sítios obtida a partir da análise elementar de nitrogênio $\left(1,46.10^{-3} \mathrm{~mol}\right.$ de sítios por grama de MMT-APTES), já que pelo ajuste não linear o valor de $\mathrm{N}_{\mathrm{s}}$ foi pouco preciso. A partir daí, impôs-se esse valor à equação de Langmuir, juntamente com os valores de $\mathrm{N}_{\mathrm{f}}$ e $\mathrm{C}_{\mathrm{eq}}$, obtendo-se valores individuais de $\mathrm{K}_{\mathrm{L}}$ para cada ponto da isoterma. A partir desses valores individuais de $\mathrm{K}_{\mathrm{L}}$ calculou-se um valor médio de $\mathrm{K}_{\mathrm{L}}$ para cada uma das isotermas nas temperaturas de 25, 35 e $45^{\circ} \mathrm{C}$. A partir dos valores médios de $\mathrm{K}_{\mathrm{L}}$ e de $\mathrm{N}_{\mathrm{s}}$, os valores de $\mathrm{N}_{\mathrm{f}}$, agora corrigidos pelo valor médio da constante, foram 
Tabela V - Valores médios de $\mathrm{K}_{\mathrm{L}}$ para adsorção de $\mathrm{Cu}^{2+}(\mathrm{aq})$ em MMT-APTES a 25,35 e $45^{\circ} \mathrm{C}$ e valor da entalpia de adsorção, $\Delta \mathrm{H}$, em $25^{\circ} \mathrm{C}$.

[Table V - Mean values of $K_{L}$ for adsorption of $\mathrm{Cu}^{2+}(a q)$ in MMT-APTES at 25, 35 and $45^{\circ} \mathrm{C}$ and the adsorption enthalpy value, $\Delta H$, at $25^{\circ} \mathrm{C}$.]

\begin{tabular}{cccc}
\hline & $25{ }^{\circ} \mathrm{C}$ & $35{ }^{\circ} \mathrm{C}$ & $45{ }^{\circ} \mathrm{C}$ \\
$* \mathrm{~K}_{\mathrm{L}}$ & $\Delta \mathrm{H}\left(\mathrm{kJ} \cdot \mathrm{mol}^{-1}\right)$ & $\mathrm{K}_{\mathrm{L}}$ & $\mathrm{K}_{\mathrm{L}}$ \\
\hline $47 \pm 10$ & $-(1,4 \pm 0,3)$ & $41 \pm 9$ & $48 \pm 6$ \\
\hline Nota: $K_{\mathrm{L}}$ foi obtido utilizando-se a concentração de equilíbrio em & mol.dm ${ }^{-3}$.
\end{tabular}

recalculados para cada ponto da isoterma a $25^{\circ} \mathrm{C} \mathrm{e}$, conforme explicado anteriormente, por aplicação da relação $\left(\Sigma \mathrm{q}_{\mathrm{R}} / \mathrm{N}_{\mathrm{f}}\right)$, obtiveram-se valores de $\Delta \mathrm{H}$ para cada ponto da titulação a 25 ${ }^{\circ} \mathrm{C}$. A Tabela $\mathrm{V}$ mostra os valores médios de $\mathrm{K}_{\mathrm{L}}$ e $\Delta \mathrm{H}$ obtidos com esse tratamento.

Os resultados obtidos mostraram que os valores das constantes de afinidade foram iguais, considerando-se as incertezas, podendo as incertezas chegarem a $21 \%$. A incerteza em $\Delta \mathrm{H}$ foi considerada igual à incerteza em $\mathrm{K}_{\mathrm{L}} \mathrm{O}$ valor de $\Delta \mathrm{H}$ foi muito pequeno, o que explica a dificuldade com a obtenção inicial de dados na calorimetria. Vale a pena comentar que o valor de $\mathrm{K}_{\mathrm{L}}$ agora obtido para $25^{\circ} \mathrm{C}, 47 \pm 10$, foi bem menor do que o valor de 273 obtido anteriormente (Tabela III), pelo ajuste não linear do modelo Langmuir. Essa diferença se justifica pelo fato de que no ajuste não linear o valor de capacidade máxima de adsorção, $\mathrm{N}_{\mathrm{s}}$, foi de 0,41 mmol. $\mathrm{g}^{-1}$ enquanto que o usado no cálculo sugerido foi de 1,46 mmol. $\mathrm{g}^{-1}$. Do ponto de vista fenomenológico, na adsorção há muitos fenômenos ocorrendo concomitantemente e de forma competitiva. A superfície e o íon $\mathrm{Cu}^{2+}$ encontravam-se hidratados; com a adsorção, essas moléculas de água foram total ou parcialmente removidas, processos endotérmicos, ao mesmo tempo que o íon $\mathrm{Cu}^{2+}$ substituiu um $\mathrm{H}^{+}$associado ao $\mathrm{NH}_{2}$ da superfície. A saída do próton é um processo endotérmico e a entrada do cobre exotérmica. A concorrência entre esses vários fenômenos é que vai determinar o sinal da entalpia. Assim, mesmo que a adsorção seja um processo muito favorável, nem sempre a componente energética é quem determina essa espontaneidade. Relembra-se que se evita aqui discutir essa questão de espontaneidade, devido à dificuldade em se estabelecer uma referência mais apropriada para a concentração (ou potencial químico). Nossa opinião concorda com a da literatura [54, 55]; não basta simplesmente optar por uma concentração em mol. $\mathrm{dm}^{-3}$ ou uma fração em mol, é preciso estabelecer isso com base na fração de superfície recoberta. Um paralelo pode-se estabelecer com a solubilidade. Quando se afirma que um processo de solubilidade é favorável numa dada condição, isso significa aproximadamente que o soluto consegue se dissolver numa concentração maior que $1 \mathrm{~mol} \cdot \mathrm{kg}^{-1}$. Para o caso da adsorção em meio condensado, seria correto tomar, por exemplo, um processo favorável como sendo aquele em que a fração de superfície recoberta seria maior que 50\% que segue o convencionado por Flory-Huggins para polímeros em solução, mas qual seria a referência para concentração, já que os valores de concentração utilizados em adsorção são muito pequenos, na faixa de ppm e ppb? Finalmente vale lembrar que o método da segunda lei da termodinâmica, embora seja correto na derivação de grandezas termodinâmicas, ele não é preciso. Os problemas surgem pelas incertezas nos valores de constantes de equilíbrio, lembrando que a energia de Gibbs guarda uma relação exponencial com a escala de constante de equilíbrio.

\section{CONCLUSÕES}

Com relação à caracterização dos materiais obtidos, os difratogramas de raios $\mathrm{X}$ mostraram que, embora $\mathrm{o}$ espaçamento basal apresentasse aumento após a intercalação com o surfactante CTAB, a organofuncionalização com APTES não ocorreu no espaço interlamelar da matriz inorgânica. Os espectros de infravermelho do material funcionalizado apresentaram os picos característicos do organossilano, o que indicou a presença do mesmo na montmorilonita. As curvas termogravimétricas do material modificado apresentaram perdas de massas atribuídas a presença do silano quimicamente ligado à estrutura do argilomineral. Após o processo de organofuncionalização os espectros de ressonância apresentaram o surgimento de um pico típico que foi atribuído à fixação do silano na estrutura da montmorilonita e, através da análise elementar, foi possível estimar a densidade de grupos imobilizados que foi 1,46 mmol. $\mathrm{g}^{-1}$. Os resultados da caracterização também mostraram que, apesar de haver um aumento do espaçamento basal da montmorilonita na intercalação do espaçador CTAB, o organossilano APTES se ligou à superfície externa da matriz inorgânica e não no seu espaço interlamelar, como se previa. Os dados de adsorção do cobre em MMT-APTES não se ajustaram bem a alguns modelos de isoterma. Com base nos dados obtidos, pôde-se inferir que a amostra funcionalizada apresentou maior eficiência na adsorção de cobre, quando comparado a alguns outros adsorventes estudados na literatura, sendo que a capacidade máxima de adsorção de $\mathrm{Cu}^{2+}$ pela montmorilonita organofuncionalizada foi cerca de 26,05 mg de $\mathrm{Cu}^{2+}$ por grama de MMT-APTES. Os resultados de isoterma não mostraram dependência com a temperatura na faixa entre 25 e $45{ }^{\circ} \mathrm{C}$. O baixo valor da entalpia de adsorção $\left(-1,4 \mathrm{~kJ} \cdot \mathrm{mol}^{-1}\right)$ evidenciou a baixa dependência da adsorção com a temperatura. Esse resultado só foi possível através da calorimetria; a sua determinação pela utilização do método baseado na segunda lei da termodinâmica, além de controverso, não foi possível nesse caso.

\section{AGRADECIMENTO}

A CAPES pelo apoio financeiro.

\section{REFERÊNCIAS}

[1] K.G. Bhattacharyya, S.S. Gupta, Colloids Surf. A 277 (2006) 191.

[2] G. Annadurai, R.S. Juang, D.J. Lee, Hazard. Mater. 92 
(2002) 263.

[3] J. Chen, A. Anandarajah, J. Geotech, Geoenviron. Eng. 128 (2002) 362.

[4] F. Wypych, K.G. Satyanarayana, Clay minerals, Elsevier, Amsterdam (2004) 553.

[5] J.V.F.L. Cavalcanti, M. da Motta, C.A.M. Abreu, O.S. Baraúna, L.A.P. Portela, Cerâmica 56 (2010) 168.

[6] Z. Sun, Y. Park, S. Zheng, G.A. Ayoko, R.L. Frost, Thermochim. Acta 569 (2013) 151.

[7] Y.C. Chiu, L.N. Huang, C.M. Uang, J.F. Huang, Colloid Surf. 46 (1990) 327.

[8] A. Reis, J.A. Simoni, A.J. Chagas, Colloid Inter. Sci. 177 (1996) 1.

[9] Y. Songyang, X. Yang, S. Xie, H. Hao, J. Song, J. Food Chem. 173 (2015) 640.

[10] Y. Yu, J.A. Mensah, D. Losic, Sci. Technol. Adv. Mater. 13 (2012) 15008.

[11] T.R. Macedo, C. Airoldi, Microporous Mesoporous Mater. 128 (2010) 158.

[12] S.P. Oliveira, W.L.L. Silva, R.R. Viana, Cerâmica 59 (2013) 338

[13] I. Langmuir, J. Am. Chem. Soc. 40 (1918) 1361.

[14] H.M.F. Freundlich, Z. Phys. Chem. 57 (1906) 385.

[15] R. Sips, J. Chem. Phys. 16 (1948) 490.

[16] C. Breen, J. Madejova, P. Komadel, J. Mater. Chem. 5 (1995) 469.

[17] C. Xu, H. Wu, F.L. Gu, J. Hazard. Mater. 275 (2014) 185.

[18] A.R.O. Júnior, Y. Pagotto, I. Valéria, M.C. Gonçalves, Acta Microsc. 12 (2003) 123.

[19] J. Venaruzzo, C. Volzone, M. Rueda, J. Ortiga, Microporous Mesoporous Mater. 56 (2002) 73.

[20] D.M. Moore, R.C. Reynolds, X-ray diffraction and identification and analysis of clay minerals, Oxford Un. Press, New York (1997) 378.

[21] J. Zhu, T. Wang, R. Zhu, F. Ge, P. Yuan, H. He, Colloids Surf. A. 384 (2011) 401.

[22] L. Su, Q. Tao, H. He, J. Zhu, P. Yuan, R. Zhu, J. Colloid Interface Sci. 391 (2013) 16.

[23] W. Hen, H. He, J. Zhu, P. Yuan, Y. Ma, X. Liang, Chinese Sci. Bull. 54 (2009) 265.

[24] M.F. Zawrah, R.M. Khattab, E.M. Saad, R.A. Gado, Spectrochim. Acta A 122 (2014) 616.

[25] M.U. de la Orden, J. Arranz, V. Lorenzo, E. Pérez, J.M. Urreaga, J. Colloid Interface Sci. 342 (2010) 185.

[26] P. Wu, Y. Dai, H. Long, N. Zhu, P. Li, J. Wu, Z. Dang, Chem. Eng. J. 191 (2012) 288.

[27] L. Gerson, L. Junior, C.W. Frederico, M.V.S. Fernandes, A.R. Loiola, Geochim. Bras. 25 (2011) 7.

[28] J. Madejová, M. Janek, P. Komadel, H.J. Herbert, H. Moog, Appl. Clay Sci. 20 (2002) 255.

[29] J. Madejová, Vib. Spectrosc. 31 (2003) 1.
[30] P.T. Bertuoli, D. Piazza, L.C. Scienza, A.J. Zattera, Appl. Clay. Sci. 87 (2014) 46.

[31] A.K. Mishra, S. Allauddin, R. Narayan, T.M Aminabhavi, K.V.S.N. Raju, Ceram. Int. 38 (2012) 929.

[32] F. Piscitelli, P. Posocco, R. Toth, M. Fermeglia, S. Pricl, G. Mensitieri, M. Lavorgna, J. Colloid Interface Sci. 351 (2010) 108

[33] A.M. Shanmugharaj, K.Y. Rhee, S.H. Ryu, J. Colloid Interface Sci. 298 (2006) 854.

[34] L. Su, Q. Tao, H. He, J. Zhu, P. Yuan, Mater. Chem. Phys. 136 (2012) 292.

[35] S.R. Ha, K.Y. Rhee, S.J. Park, J.H. Lee, Compos. Part B Eng. 41 (2010) 602.

[36] A.A. Silva, K. Dahmouche, B.G. Soares, Appl. Clay Sci. 54 (2011) 151.

[37] Y. Park, G.A. Ayoko, J. Kristof, E. Horváth, R.L. Frost, J. Therm. Anal. Calorim. 107 (2012) 1137.

[38] Y. Xi, Z. Ding, H. He, R.L. Frost, J. Colloid Interface Sci. 277 (2004) 116.

[39] Y. Park, G.A. Ayoko, J. Kristof, E. Horváth, R.L. Frost, J. Therm. Anal. Calorim. 110 (2012) 1087.

[40] Y. Xi, R.L. Frost, H. He, T. Kloprogge, T. Bostrom, Langmuir 21 (2005) 8675.

[41] J.Y. Lee, H.K. Lee, Mater. Chem. Phys. 85 (2004) 410. [42] M. Alshabanat, A. Al-Arrash, W. Mekhamer, J. Nanomater. 2013 (2013) 1.

[43] Z. Gu, M. Gao, L. Lu, Y. Liu, S. Yang, Ind. Eng. Chem. Res. 54 (2015) 4947.

[44] P.T. Bertuoli, D. Piazza, L.C. Scienza, A.J. Zattera, Appl. Clay Sci. 87 (2014) 46.

[45] H. He, J. Duchet, J. Galy, J.F. Gerard, J. Colloid Interface Sci. 288 (2005) 171.

[46] W. Shen, H.P. He, J. Zhu, P. Yuan, R.L. Frost, J. Colloid Interface Sci. 313 (2007) 268.

[47] C.H. Rhee, H.K. Kim, H. Chang, J.S. Lee, Chem. Mater. 17 (2005) 1691.

[48] P.H. Thiesen, K. Beneke, G. Lagaly, J. Mater. Chem. 12 (2002) 3010

[49] A. Dąbrowski, Adv. Colloid Interface Sci. 93 (2001) 135.

[50] H. Aydin, Y. Bulut, Ç. Yerlikaya, J. Environ. Manage. 87 (2008) 37.

[51] L. Ma, Q. Chen, J. Zhu, Y. Xi, H. He, R. Zhu, Q. Tao, G.A. Ayoko, Chem. Eng. J. 283 (2016) 880.

[52] M. Fouladgar, M. Beheshti, H. Sabzyan, J. Mol. Liq. 211 (2015) 1060.

[53] O. Abollino, M. Aceto, M. Malandrino, C. Sarzanini, E. Mentasti, Water Res. 37 (2003) 1619.

[54] Y. Chang, J.Y. Lai, D.J. Lee, Bioresour. Technol. 222 (2016) 513

[55] I. Anastopoulos, G.Z. Kyzas, J. Mol. Liq. 218 (2016) 174 .

(Rec. 24/11/2017, Rev. 18/01/2018, Ac.07/02/2018) 